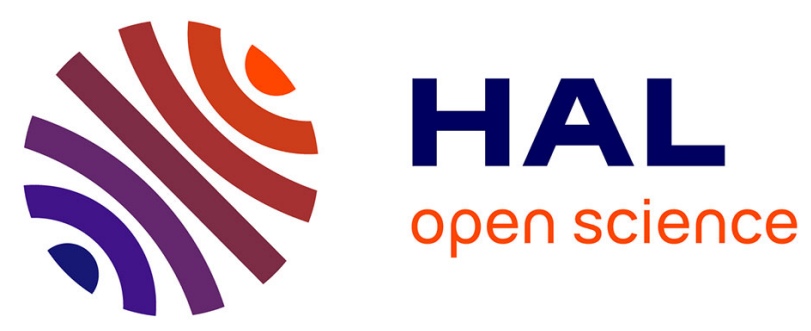

\title{
Statistical Analysis of Non-Deterministic Fork-Join Processes
}

\author{
Antoine Genitrini, Martin Pépin, Frédéric Peschanski
}

\section{To cite this version:}

Antoine Genitrini, Martin Pépin, Frédéric Peschanski. Statistical Analysis of Non-Deterministic ForkJoin Processes. Theoretical Aspects of Computing - ICTAC 2020 - 17th International Colloquium, Nov 2020, Macau, China. pp.83-102, 10.1007/978-3-030-64276-1_5 . hal-02659801v2

\section{HAL Id: hal-02659801 \\ https://hal.sorbonne-universite.fr/hal-02659801v2}

Submitted on 21 Oct 2020

HAL is a multi-disciplinary open access archive for the deposit and dissemination of scientific research documents, whether they are published or not. The documents may come from teaching and research institutions in France or abroad, or from public or private research centers.
L'archive ouverte pluridisciplinaire HAL, est destinée au dépôt et à la diffusion de documents scientifiques de niveau recherche, publiés ou non, émanant des établissements d'enseignement et de recherche français ou étrangers, des laboratoires publics ou privés. 


\title{
Statistical Analysis of Non-Deterministic Fork-Join Processes ${ }^{\star}$
}

\author{
Antoine Genitrini ${ }^{[0000-0002-5480-0236]}$, Martin Pépin ${ }^{[0000-0003-1892-3017]}$, and \\ Frédéric Peschanski ${ }^{[0000-0002-4206-3283]}$ \\ Sorbonne Université, CNRS, LIP6, UMR7606, F-75005 Paris, France. \\ \{antoine.genitrini, martin.pepin, frederic.peschanski\}@lip6.fr
}

\begin{abstract}
We study the combinatorial structure of concurrent programs with non-deterministic choice and a fork-join style of coordination. As a first step we establish a link between these concurrent programs and a class of combinatorial structures. Based on this combinatorial interpretation, we develop and experiment algorithms aimed at the statistical exploration of the state-space of programs. The first algorithm is a uniform random sampler of bounded executions, providing a suitable default exploration strategy. The second algorithm is a random sampler of execution prefixes that allows to control the exploration with respect to the uniform distribution. The fundamental characteristic of these algorithms is that they work on the control graph of the programs and not directly on their state-space, thus providing a way to tackle the state explosion problem.
\end{abstract}

Keywords: Concurrency · Non-Determinism • Fork-Join Processes · Loops · Combinatorics · Uniform Random Generation

\section{Introduction}

Analyzing the state-space of concurrent programs is a notoriously difficult task, if only because of the infamous state explosion problem. Several techniques have been developed to "fight" this explosion: symbolic encoding of the state-space, partial order reductions, exploiting symmetries, etc. An alternative approach is to adopt a probabilistic point of view, for example by developing statistical analysis techniques such as 14. The basic idea is to generate random executions from program descriptions, sacrificing exhaustiveness for the sake of tractability. However, there is an important difference between generating an arbitrary execution and generating a random execution according to a known (typically the uniform) distribution. Only the latter allows to estimate the coverage of the state-space of a given analysis.

As a preliminary, we have to find suitable combinatorial interpretations for the fundamental constructions of concurrent programs. In this paper, we study

* This research was partially supported by the ANR MetACOnc project ANR-15CE40-0014. 
a class of programs that uses a fork-join model of synchronization, together with loops and a choice construct for non-determinism. This is a simple formalism but it is non-trivial in terms of the concurrency features it provides. Most importantly, the underlying combinatorial interpretation is already quite involved. In previous work we studied the combinatorial interpretation of three fundamental aspects of concurrency: parallelism (as interleaving) interpreted as (strictly) increasing labelled structures [8, non-determinism as partial labelling [7] and synchronization as non-strict labelling [9]. In this paper, we integrate these various interpretations into a single unified combinatorial specification based on the symbolic method of [2]. The main interest of this specification of process behaviors is that we can then obtain, in a systematic way, the generating function for the possible executions of a given program. Because we only study finite objects, the executions are considered of bounded length. At the theoretical level, this is often a suitable starting point for the study of a quantitative problem in analytic combinatorics (cf. e.g. [8]). At a more practical level, the combinatorial specification is also a good source of algorithmic investigations, which is our main concern in the present paper.

The first problem we study is that of counting the number of executions (of bounded length) of the programs. This is the problem one has to tackle to precisely quantify the so-called state "explosion", and it is also an important building block of our algorithmic toolbox. Unfortunately, counting executions of concurrent programs is in fact hard in the general case. We show in 9 that even for simple programs only allowing barrier synchronization, counting executions is a $\sharp P$-complete problem ${ }^{1}$ Fork-join parallelism enables a good balance between tractability and expressivity by enforcing some structure in the state-space. A second problem is caused by non-determinism because for each non-deterministic choice we have to select a unique branch of execution. Moreover, choices can be nested so that the number of possibilities can grow exponentially. Relying on an efficient encoding of the state-space as generating functions, we manage to count executions without expanding the choices. Of course counting executions has no direct practical application, but it is an essential requirement for two complementary and more interesting analysis techniques. First, we develop an efficient algorithm to generate executions of a given process uniformly at random, for a given bounded execution length. Without prior knowledge of the state-space, the uniform distribution yields the best coverage, with the best diversity of outputs. The second algorithm generates random prefixes, which allows the user to introduce some bias in the statistical exploration strategy, e.g. towards regions of interest of the state-space, while still giving a good coverage and most importantly still giving control over the distribution. A fundamental characteristics of these algorithms is that they work on the syntactic representation of the program and do not require the explicit construction of the state-space, hence enabling the analysis of systems of a rather large size.

\footnotetext{
${ }^{1}$ A function $f$ is in $\sharp P$ if there is a polynomial-time non-deterministic Turing machine $M$ such that for any instance $x, f(x)$ is the number of executions of $M$ that accept $x$ as input 23 .
} 
The outline of the paper is as follows. In Section 2 we present the program class of non-deterministic fork-join programs, as well as its combinatorial interpretation. In Section 3 we present in details the two random sampling algorithms discussed above. Finally, Section 4 provides a preliminary experimental study of the algorithm ${ }^{2}$.

Related work Our study combines viewpoints and techniques from concurrency theory and combinatorics. A similar line of work exists for the so-called "true concurrency" model (by opposition to the interleaving semantics that we use in our study) based on the trace monoid using heaps combinatorics (see [181]). To our knowledge these only address the parallelism issue and not non-determinism per se. In [3, the authors cover the problem of the uniform random generation of words in a class of synchronised automata. This approach is able to cover a slightly more expressive set of programs but this comes at the cost of the construction of a product (synchronizing) automaton of exponential size in the worst case. Another approach, investigated in the context of MonteCarlo model-checking, is based on the combinatorics of lassos, which relates to the verification of some temporal-logic properties over potentially infinite executions. In [15, the authors of this method highlight the importance of uniformity. Later 21] gives a uniform random sampler of lassos, however relying on the costly explicit construction of the whole state-space, hence unpractical for even small processes. Finally [10] studies the random generation of executions in a model similar to the one we cover by extending the framework of Boltzmann sampling. Although Boltzmann samplers are usually fast, they turn out to be impractical in this context because of the heavy symbolic computations imposed by the interplay between parallelism and synchronisation.

If compared to [7, which discusses non-determinism without synchronization, we adopt in the present paper a more direct and simpler encoding of nondeterministic choices, which significantly improves both the theoretical and practical developments. The algorithms presented in the paper are in consequence much more efficient in practice, while covering a more expressive language.

\section{Non-deterministic fork-join programs with loops}

We introduce in this section a simple class of concurrent programs featuring a fork-join programming style with non-deterministic choices and loops. The interest is twofold. First it showcases non-determinism in interaction with a non-trivial programming model, which gives insights about its quantitative and algorithmic aspects. Second the language supports a simple model of iteration, for which we give a combinatorial interpretation. In terms of expressivity, this is an important step forward compared to our previous work (see [8/7/5 for instance). Throughout the paper we will refer to this class as the class of nondeterministic fork-join programs.

\footnotetext{
${ }^{2}$ An implementation of our algorithms and all the scripts used for the experiments can be found on the companion repository at https://gitlab.com/ParComb/libnfj.
} 


\subsection{Syntax}

Definition 1 (Non-deterministic fork-join programs). Given a set of symbols $\mathcal{A}$ representing the "atomic actions" of the language, the class of nondeterministic fork-join programs (over this set $\mathcal{A}$ ), denoted NFJ, is defined as follows:

$$
\begin{aligned}
& P, Q \quad:=P \| Q \quad \text { (parallel composition (or fork)) } \\
& \mid P ; Q \quad \text { (sequential composition (or join)) } \\
& P+Q \quad \text { (non-deterministic choice) } \\
& P^{\star} \quad \text { (loop) } \\
& a \in \mathcal{A} \quad \text { (atomic action) } \\
& \text { | } 0 \text { (empty program, noop). }
\end{aligned}
$$

Informally, the first two constructions form the fork-join "core" of the language: $P \| Q$ expresses the fact that $P$ is run in parallel with $Q$ and $P ; Q$ means that $P$ must terminate before $Q$ starts. In $a ;(b \|(c ; d)) ; e$, the program starts by firing $a$, then it forks two processes $b$ and $c ; d$ which run in parallel and when they terminate $e$ is run, which is called a "join". The third construction $P+Q$ expresses a choice: either $P$ or $Q$ is executed but not both. This can model an "internal" choice of the system such as a random event, a system failure etc, or an "external" choice, that is a choice depending on a user input 3 . Finally, the construction $P^{\star}$ expresses loops that can have any (finite) number of iterations. For instance $(a ;(b \| c))^{\star}$ can be unrolled at runtime to 0 (zero iteration), $a ;(b \| c)$ (one iterations), $a ;(b \| c) ; a ;(b \| c)$ (two iterations), etc.

It is important to mention now that the nature of the atomic actions will remain abstract in the present work, we treat them as black boxes and will consider that the different occurrences of an action across a term are distinct. These are sometimes referred to as events in the literature. Our focus is set on the order in which these actions can be fired and scheduled by the different operators of the language. In all our examples we use a different lowercase roman letters as a unique identifiers to help distinguishing between each action.

This simple model is expressive enough to write simple programs in the forkjoin style. Moreover, the four combinators present in the grammar above can be modelled and well-understood using the tools from analytic combinatorics, which is at the core of our random sampling procedures in Section 3 .

\subsection{Semantics}

We give NFJ an operational semantics in the style of [17. We define a "reduction" relation $P \stackrel{a}{\rightarrow} P^{\prime}$ between two programs and an atomic action, it reads "program $P$ reduces to $P^{\prime}$ by firing action $a$ ". The idea behind the rules is explained just below.

\footnotetext{
${ }^{3}$ Since we do not interpret the action symbols, no distinction is possible in the provided semantics between a choice being triggered internally or externally.
} 


$$
\begin{array}{ccc}
\frac{P \stackrel{a}{\rightarrow} P^{\prime}}{P\left\|Q \stackrel{a}{\rightarrow} P^{\prime}\right\| Q} \text { (Lpar) } & \frac{Q \stackrel{a}{\rightarrow} Q^{\prime}}{P\|Q \stackrel{a}{\rightarrow} P\| Q^{\prime}} \text { (Rpar) } & \frac{P \stackrel{a}{\rightarrow} P^{\prime}}{P ; Q \stackrel{a}{\rightarrow} P^{\prime} ; Q} \text { (Lseq) } \\
\frac{\text { nullable }(P) \quad Q \stackrel{a}{\rightarrow} Q^{\prime}}{P ; Q \stackrel{a}{\rightarrow} Q^{\prime}} \text { (Rseq) } & \left.\frac{P \stackrel{a}{\rightarrow} P^{\prime}}{P+Q \stackrel{a}{\rightarrow} P^{\prime}} \text { (Lchoice) }\right) & \frac{Q \stackrel{a}{\rightarrow} Q^{\prime}}{P+Q \stackrel{a}{\rightarrow} Q^{\prime}} \text { (Rchoice) } \\
\left.\frac{}{a \stackrel{a}{\rightarrow} 0} \text { (act) } \frac{P \stackrel{a}{\rightarrow} P^{\prime}}{P^{\star} \stackrel{a}{\rightarrow} P^{\prime} ; P^{\star}} \text { (loop }\right)
\end{array}
$$

The nullable predicate, defined just below, tells whether a program can terminate without firing any action.

$$
\begin{array}{ll}
\operatorname{nullable}(P \| Q)=\operatorname{nullable}(P) \wedge \operatorname{nullable}(Q) & \text { nullable }(0)=\top \\
\text { nullable }(P ; Q)=\operatorname{nullable}(P) \wedge \operatorname{nullable}(Q) & \text { nullable }(a)=\perp \\
\text { nullable }(P+Q)=\operatorname{nullable}(P) \vee \operatorname{nullable}(Q) & \text { nullable }\left(P^{\star}\right)=\top
\end{array}
$$

The rules for the parallel composition (Lpar and Rpar) express the interleaving semantics of the language: if an action can be fired in any of $P$ or $Q$, then it can be fired in $P \| Q$ and the term is rewritten. By iterating these two rules, we can obtain any interleaving of an execution of $P$ and an execution of $Q$. Sequential composition is more asymmetric. The Lseq rule is similar to Lpar but Rseq captures the synchronisation: an execution can be fired on the right-hand-side only if the left-hand-side is ready to terminate (expressed by nullable $(P)$ ), in which case it is erased. The choice rules Lchoice and Rchoice allow actions to be fired from both sides but once we have made the choice of the branch, it is made definitive by erasing the other branch. Finally the loop $P^{\star}$ can be unrolled any number of times, which is expressed by giving $P^{\star}$ the same semantics as $0+\left(P ; P^{\star}\right)$. The fact that nullable $P^{\star}$ holds expresses that the loop can be unrolled zero times, and thus behave as the 0 program.

We call "execution step" a proof-tree built from the above rules (and not simply its conclusion) and we define an execution as a sequence of such steps leading to a nullable term.

Definition 2 (Execution). An execution of an NFJ program $P_{0}$ is a sequence of steps of the form $P_{0} \stackrel{a_{1}}{\Rightarrow} P_{1} \stackrel{a_{2}}{\Rightarrow} P_{2} \ldots \stackrel{a_{n}}{\Rightarrow} P_{n}$, such that nullable $\left(P_{n}\right)$ holds, and where for all $i, P_{i-1} \stackrel{a_{i}}{\Rightarrow} P_{i}$ is a proof-tree ending on the conclusion $P_{i-1} \stackrel{a_{i}}{\rightarrow} P_{i}$.

We refer to the set of all possible executions of a program as its state-space.

Remark 1 (on equality). We purposely based our notion of execution on the proof-trees rather than simply on the relation $P \stackrel{a}{\rightarrow} P^{\prime}$ to capture the choices hidden inside these steps. For instance there are two distinct executions depicted by $a^{\star \star} \stackrel{a}{\Rightarrow}\left(0 ; a^{\star} ; a^{\star \star}\right) \stackrel{a}{\Rightarrow}\left(0 ; a^{\star} ; a^{\star \star}\right)$. One corresponds to the case where the 
outer loop is only unrolled once (i.e. the (loop) rule is applied once) but the inner loop twice. The other corresponds to the case where the outer loop is unrolled twice and the two occurrences of the inner loop once. The reason behind this choice is that we focus on the control-flow of programs here rather than the actual content of the atomic actions.

We will take the following program as a running example for the rest of the paper: $P_{0}=\left((a+(b \| c))^{\star} \|(d+0)\right)^{\star} ;(e+(f \| g))$. This program has one length-1 execution, with the following proof tree:

$$
\frac{\text { nullable }\left(\left((a+(b \| c))^{\star} \|(d+0)\right)^{\star}\right) \frac{\overline{e \stackrel{e}{\rightarrow} 0}(\text { act })}{e+(f \| g) \stackrel{e}{\rightarrow} 0}(\text { Lchoice })}{P_{0} \stackrel{e}{\rightarrow} 0}(\text { Rseq })
$$

There are also four length-2 executions, as follow: 4

$-P_{0} \stackrel{f}{\Rightarrow}(0 \| g) \stackrel{g}{\Rightarrow} 0 \| 0$

$-P_{0} \stackrel{g}{\Rightarrow}(f \| 0) \stackrel{f}{\Rightarrow} 0 \| 0$

- $P_{0} \stackrel{a}{\Rightarrow}\left(\left(0 ;(a+(b \| c))^{\star}\right) \|(d+0)\right) ;\left((a+(b \| c))^{\star} \|(d+0)\right)^{\star} ;(e+(f \| g)) \stackrel{e}{\Rightarrow} 0$

$-P_{0} \stackrel{d}{\Rightarrow}\left((a+(b \| c))^{\star} \| 0\right) ;\left((a+(b \| c))^{\star} \|(d+0)\right)^{\star} ;(e+(f \| g)) \stackrel{e}{\Rightarrow} 0$.

\subsection{Combinatorial interpretation}

We now give an interpretation of the executions of an NFJ program as combinatorial objects, which will open us the toolbox of analytic combinatorics for the rest of the paper. We model the set of the executions of a program as a combinatorial class using the formalism from [12, which we recall here. A combinatorial class is a potentially infinite set of objects where each object has been given a (finite) size and in which there is only a finite number of objects of each size. In our case, the combinatorial class of interest is the set of finite executions of a given program and the size of an execution is its length i.e. its number of reduction steps.

The combinatorial class $S(P)$ modelling the executions of $P$ is inductively defined in Table 1. The explanations for the combinatorial constructions are given bellow.

The empty program 0 and the atomic action $a$ have only one execution, of length 0 and 1 respectively. This is modelled combinatorially by the neutral class $\mathcal{E}$ : the class containing only one element of size 0 , and the atom class $\mathcal{Z}$ : the class with only one element of size 1 .

\footnotetext{
${ }^{4}$ In general, the notation $P \stackrel{a}{\Rightarrow} P^{\prime}$ is ambiguous since there may be several different proof-trees with the same conclusion $P \stackrel{a}{\rightarrow} P^{\prime}$. An example of this is given in Remark 1. For the sake of simplicity and in order to keep the notations light, in the examples given here, each step $P \stackrel{a}{\Rightarrow} P^{\prime}$ identifies only one possible proof-tree.
} 
Table 1. Recursive rules for the computation of the generating function of executions of an NFJ program.

\begin{tabular}{ll}
\hline Construction & Specification \\
\hline$P$ & $S(P)$ \\
\hline 0 & $\mathcal{E}$ \\
$a$ & $\mathcal{Z}$ \\
$P \| Q$ & $S(P) \star S(Q)$ \\
$P ; Q$ & $S(P) \times S(Q)$ \\
$P+Q$ when nullable $(P) \wedge$ nullable $(Q)$ & $S(P)+(S(Q) \backslash \mathcal{E})$ \\
$P+Q$ otherwise & $S(P)+S(Q)$ \\
$P^{\star}$ when nullable $(P)$ & $\operatorname{SEQ}(S(P) \backslash \mathcal{E})$ \\
$P^{\star}$ otherwise & $\operatorname{SEQ}(S(P))$ \\
\hline
\end{tabular}

The first interesting case is the parallel composition: the executions of $P \| Q$ are made of any interleaving of one execution of $P$ and one execution of $Q$. For instance if $P=a+(b ; c)$ and $Q=d^{\star}$, then $P$ admits for instance an execution firing $b$ and then $c$ (denoted by $b c$ for short) and $Q$ admits an execution firing two $d$ s (denoted by $d d$ for short). Then all the 6 possible interleavings of these executions are executions of $P \| Q: b c d d, b d c d, b d d c, d b c d, d b d c$ and $d d b c$ (again, we only denote the executions by their firing sequences for conciseness). The labelled product ${ }^{5} \mathrm{bf}$ combinatorics expresses exactly this and is denoted using the $\star$ symbol. The executions of $P$; $Q$ are given by an execution of $P$ followed by an execution of $Q$. So for instance, using the same example programs $P$ and $Q$ as above, $b c d d$ is an execution of $(P ; Q)$ but not $d b c d$. So they can be seen as a pair of an execution of $P$ and an execution of $Q$ which is naturally modelled using the Cartesian product. The set of executions of $P+Q$ is the union of the executions of $P$ and $Q$. Moreover this union is "almost" disjoint in the sense that the only execution that these programs may have in common is the empty execution, hence the two cases in the definition. Combinatorially, the fact that nullable $(P)$ holds corresponds to the fact that the class of its executions contains one object of size 0 : the empty execution. It is in fact important that we can express this in terms of disjoint unions because they fit in the framework of analytic combinatorics whereas arbitrary unions are more difficult to handle ${ }^{6}$

Finally, the executions of $P^{\star}$ are sequences of executions of $P$ or, equivalently, sequences of non-empty executions of $P$. This second formulation leads to a non-ambiguous specification as the unique class $\mathcal{P}^{\prime}$ satisfying $\mathcal{P}^{\prime}=\mathcal{E}+\mathcal{P}_{+} \times \mathcal{P}^{\prime}$, where $\mathcal{P}_{+}$denotes the non-empty executions of $P$. This implicitly defined class $\mathcal{P}^{\prime}$

\footnotetext{
5 The word "labelled" is not particularly relevant in our setup. It refers to the fact that another way to represent the interleaving of two executions is to put an integer label on each step of both execution carrying the position of each step in the interleaving.

${ }^{6}$ Grammar descriptions involving non-disjoint unions are referred to as "ambiguous" and lack most of the benefits, if not all, of the symbolic method, essentially because some objects may be counted multiple times when applying the method.
} 
Table 2. The rules of the symbolic method for computing a generating function from a combinatorial specification. In the case of the labelled product $\mathcal{A} \star \mathcal{B}$, the corresponding operation on the series is called the coloured product $\odot$ and is defined in [6] by $A(z) \odot$ $B(z)=\sum_{n \geqslant 0} \sum_{k=0}^{n}\left(\begin{array}{l}n \\ k\end{array}\right) a_{k} b_{n-k} z^{n}$.

\begin{tabular}{ll}
\hline Specification & Gen. Function \\
$\mathcal{A}$ & $A(z)$ \\
\hline $\mathcal{E}$ & 1 \\
$\mathcal{Z}$ & $z$ \\
$\mathcal{A} \backslash \mathcal{B}$ (only when $\mathcal{B} \subset \mathcal{A})$ & $A(z)-B(z)$ \\
$\mathcal{A}+\mathcal{B}$ & $A(z)+B(z)$ \\
$\mathcal{A} \star \mathcal{B}$ & $A(z) \odot B(z)$ \\
$\mathcal{A} \times \mathcal{B}$ & $A(z) \cdot B(z)$ \\
$\operatorname{SEQ}(\mathcal{A})$ & $(1-A(z))^{-1}$ \\
\hline
\end{tabular}

is denoted $\operatorname{SEQ}\left(\mathcal{P}_{+}\right)$and is called the sequence of $\mathcal{P}_{+}$. Once again we must distinguish whether nullable $(P)$ holds or not in the definition of $\mathcal{P}_{+}$to avoid ambiguities and thus double-counting.

The $S$ function described above maps each program to a combinatorial specification of its executions. As an example, for our example program we have $S\left(P_{0}\right)=\operatorname{Seq}(\operatorname{Seq}(\mathcal{Z}+(\mathcal{Z} \star \mathcal{Z})) \star(\mathcal{Z}+\mathcal{E}) \backslash \mathcal{E}) \times(\mathcal{Z}+(\mathcal{Z} \star \mathcal{Z}))$. Such a specification is often the starting point of the study of a problem in analytic combinatorics, because it has many outcomes, and one of the most important of them is that it gives a systematic way to compute the generating function of the combinatorial class. We recall that the generating function of a class $\mathcal{C}$ is the formal power series given by $C(z)=\sum_{n \geqslant 0} c_{n} z^{n}$ where $c_{n}$ is the number of elements of size $n$ in $\mathcal{C}$.

The generating function of the executions of a program, i.e. of the class $S(P)$, constitutes a summary of the counting information of its state space. Moreover, this encoding as a power series gives a convenient formalism to compute the number of executions of length $n$, for bounded $n$. The symbolic method from [12] gives an automatic translation from the specification of a class to its generating function, which we recall in Table 2 .

We now illustrate the power of the analytic combinatorics tools, by showing how a few manipulations on polynomials can lead to interesting algorithmic applications and precise quantitative results. Further resource on this topic can be found in the book [12. We study the generating function $\phi$ of the example program $P_{0}$ given above, which we recall here for convenience: $P_{0}=$ $\left[(a+(b \| c))^{\star} \|(d+0)\right]^{\star} ;[e+(f \| g)]$. Let $\phi(z)=\sum_{n \geqslant 0} p_{n} z^{n}$ denote the expansion in power series of the generating function of $S\left(P_{0}\right)$ and recall that the $n$-th coefficient $p_{n}$ is the number of executions of $P_{0}$ of length $n$. By applying the 
rules from Table 2 to $S\left(P_{0}\right)$ we obtain that:

$$
\begin{aligned}
\phi(z) & =\left[\left(1-z-2 z^{2}\right)^{-1} \odot(z+1)\right]^{-1} \cdot\left[z+2 z^{2}\right] \\
& =\frac{(2 z+1)(2 z-1)^{2}(z+1)^{2} z}{1-4 z-4 z^{2}+6 z^{3}+8 z^{4}}
\end{aligned}
$$

The second line of the above formula is obtained by applying the calculus $\operatorname{rul} 7^{7} z \odot A(z)=z \frac{d(z A(z))}{d z}$. From this formula we derive two applications. First, from the denominator of this rational expression we deduce that for all $n>6$ we have $p_{n}-4 p_{n-1}-4 p_{n-2}+6 p_{n-3}+8 p_{n-4}=0$. The obtained recurrence formula can be used to compute the number of executions of length $n$ of $P_{0}$ in linear time. On the analytic side, $\phi$ being a rational function, we can do a partial fraction decomposition to obtain $\phi$ as a sum of four terms of the form $C_{i}\left(1-z \rho_{i}^{-1}\right)$ (plus a polynomial). Each of these terms expands as $\sum_{n \geqslant 0} C_{i} \rho_{i}^{-n} z^{n}$, hence the number of executions of $P_{0}$ of length $n$ satisfies $p_{n}=C \cdot \rho^{-n} \cdot(1+o(1))$ for some constants $C$ and $\rho$ and with an exponentially small error term hidden in the $o(1)$. In this case we have $\rho \approx 0.221987, C \approx 0.146871$ and the error term is of the order of $0.327950^{n}$. Table 3 compares the values of $p_{n}$ and of the proposed approximation for a few values of $n$. One can see that already for small values of $n$, the relative error of this approximation is rather low.

Table 3. Value of $p_{n}$, of its approximation $C \cdot \rho^{-n}$ and of the relative error $\mid p_{n}-C$. $\rho^{-n} \mid / p_{n}$ for small values of $n$.

\begin{tabular}{lrrrrrrr}
\hline$n$ & 6 & 7 & 8 & 9 & 10 & 11 & 12 \\
\hline$p_{n}$ & 1226 & 5528 & 24904 & 112196 & 505424 & 2276832 & 10256616 \\
approx & 1227 & 5529 & 24907 & 112199 & 505429 & 2276839 & 10256626 \\
rel. err. & 0.000816 & 0.000181 & 0.00012 & $2.67 \mathrm{e}-05$ & $9.89 \mathrm{e}-06$ & $3.07 \mathrm{e}-06$ & $9.75 \mathrm{e}-07$ \\
\hline \multicolumn{7}{r}{30} \\
\hline 20 & 30 & & 50 \\
\hline 1739330569856 & 5985551205783341568 & 70883995824212596666294027026432 \\
1739330570089 & 5985551205783353055 & 70883995824212596666294055205537 \\
$1.34 \mathrm{e}-10$ & $1.92 \mathrm{e}-15$ & & $3.98 \mathrm{e}-25$ \\
\hline
\end{tabular}

\section{Statistical analysis algorithms}

In this section, we study the problem of exploring the state-space of a given process through random generation. We describe first a uniform random sampler

\footnotetext{
7 This is the only "non-standard" computation rule we use in this example. All the rest is usual polynomial manipulations. General rules for computing $A(z) \odot B(z)$ are beyond the scope of this article.
} 
of executions of given length, and second a uniform random sampler of execution prefixes. Our approach relies on the counting information contained in the generating functions, as defined previously.

\subsection{Preprocessing: the generating function of executions}

As explained in the previous section, the symbolic method gives a systematic way of computing the generating function of the class of the executions of a program $P$ from its specification $S(P)$ using the rules from Table 2 . A straightforward application of this method leads to Algorithm 1 for computing the first terms of the series.

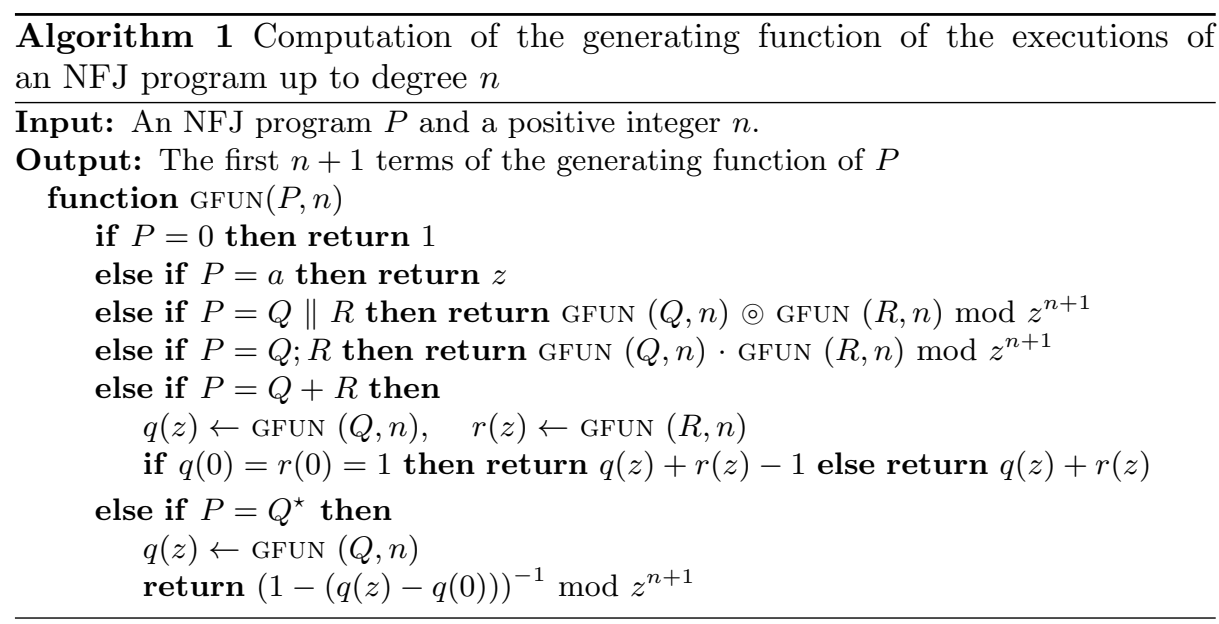

The coloured product $\odot$ used in the parallel composition case can be implemented using the formula $A(z) \odot B(z)=\operatorname{Lap}(\operatorname{Bor}(A) \cdot \operatorname{Bor}(B))$ where Bor and Lap are respectively the combinatorial Borel and Laplace transforms (see [6] for insights on the coloured product). This approach has the advantage of benefiting from the efficient polynomial multiplication algorithms from the literature at the cost of three linear transformations. To be implemented efficiently, the coefficients of the result of the Borel transform should share $n$ ! as a common denominator so that it is only stored once and we keep working with integer coefficients. The computation of $(1-A(z))^{-1}$ can be carried out efficiently using Newton iteration (see 22] for instance). The idea is to iterate the formula $S_{i+1}(z) \leftarrow S_{i}(z)+S_{i}(z) \cdot\left(A(s) \cdot S_{i}(z)-\left(S_{i}(z)-1\right)\right)$, starting from $S_{0}(z)=1$. It has been shown that only $\left\lceil\log _{2}(n+1)\right\rceil$ iterations are necessary for the coefficients of $S_{i}(z)$ to be equal to those of $(1-A(z))^{-1}$ up to degree $n$. Moreover the total cost of this procedure in terms of integer multiplication is of the same order of magnitude as that of the multiplication of two polynomials of degree $n$. 
Theorem 1. Let $P$ be an NFJ program and let $|P|$ denote its syntactic size (i.e. the number of constructors $\|,+, ;,{ }^{\star}$ and atomic actions) in its definition). Algorithm 1 can be implemented to compute the first $n$ coefficients of the generating function of the executions of $P$ in $O(|P| M(n))$ operations on big integers where $M(n)$ is the complexity of the multiplication of two polynomials of degree $n$. Moreover, these coefficients are bounded by $n$ ! and hence have at most $n \log _{2}(n)$ bits.

Proof. The proof of Theorem 1 follows from the above discussion: each constructor incurs one polynomial operation among addition, multiplication, coloured product and inversion and all of them can be carried out in $O(M(n))$.

To give a rough idea of the performance that can be achieved by Algorithm 1 . we computed the generating function of $P_{0}$ up to degree $n=10000$ - and thus its number of executions of length $k$ for all $k \leqslant 10000$ - in less that 4 s on a standard PC. A detailed benchmark of Algorithm 1 is given in Section 4.1

\subsection{Random sampling of executions}

Another consequence of having a combinatorial specification of the state-space at our disposal is that we can apply well-known random sampling methods from the combinatorics toolbox. Our random sampling procedure for program executions is based on the so-called "recursive-method" from [13. It operates in a similar fashion to the symbolic method, that is by induction on the specification by combining the random samplers of the sub-structures with simple rules depending on the grammar construction. For the sake of clarity we represent executions as sequences of atomic actions. This encoding does not contain all the information that defines an execution, typically it does not reflect in which iteration of a loop an atomic action is fired for instance. However it makes the presentation clearer and the algorithm can be easily adapted to a more faithful encoding. Our uniform random sampler of executions is described in Algorithm 2 and the detailed explanations about the different constructions are given below.

Choice The simplest rule of the recursive method is that of the disjoint union used at line 4 of Algorithm 2. If $q_{n}$ and $r_{n}$ denote the number of length- $n$ executions of $Q$ and $R$, then a uniform random length- $n$ execution of $P=Q+R$ is a uniform length- $n$ execution of $Q$ with probability $q_{n} /\left(q_{n}+r_{n}\right)$ and a uniform length- $n$ execution of $R$ otherwise. One way to draw the Bernoulli variable is to draw a uniform random big integer $x$ in $\llbracket 0 ; q_{n}+r_{n} \llbracket$ and to return true if and only if $x<q_{n}$. As an example, consider the programs $Q=(a+(b \| c))$ and $R=d^{\star}$. We count that $Q$ has two executions of length two: $b c$ and $c b$ and $R$ has only one: $d d$. Hence, to sample a length-2 execution in $(Q+R)$, one must perform a recursive call on $Q$ with probability $2 / 3$ and on $R$ with probability $1 / 3$.

Parallel composition The other rules build on top of the disjoint union case. For instance, the set of length- $n$ executions of $P=Q \| R$ can be seen as $\mathcal{Q}_{0} \star \mathcal{R}_{n}+\mathcal{Q}_{1}$ * 


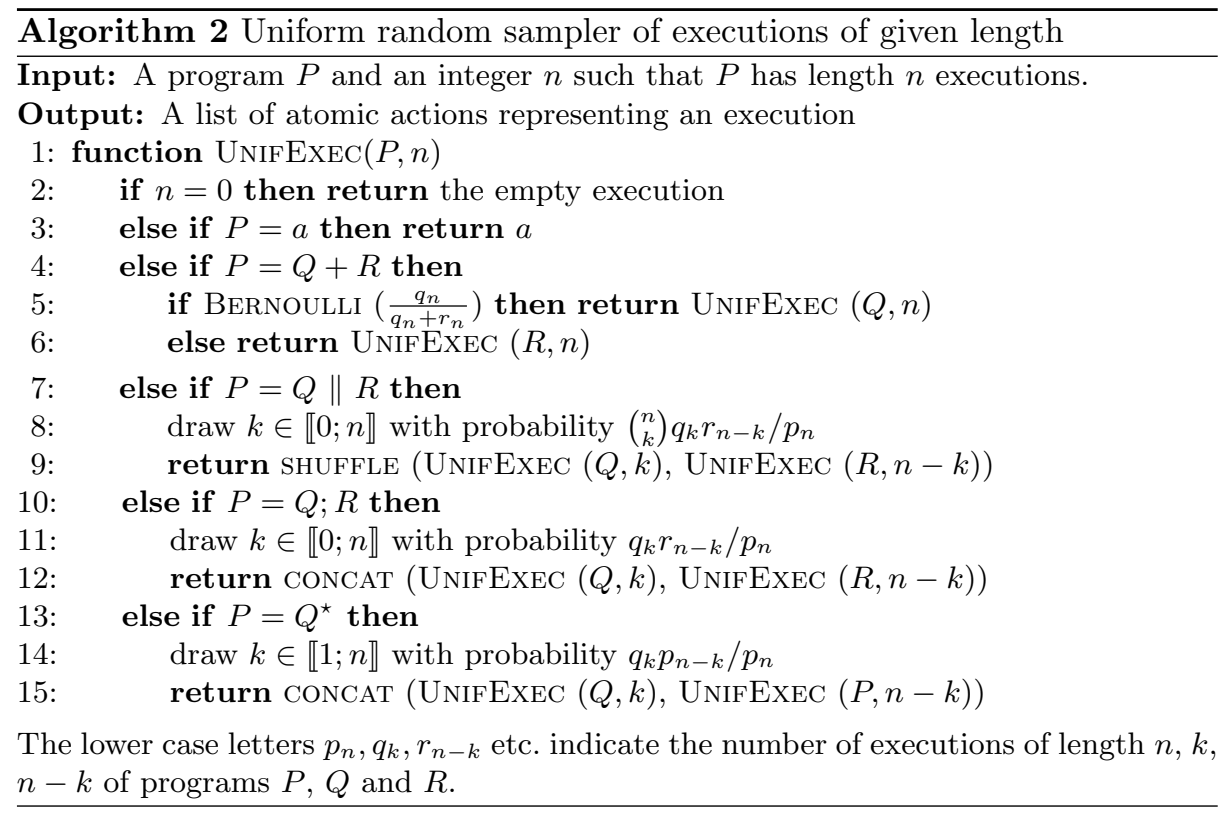

$\mathcal{R}_{n-1}+\cdots+\mathcal{Q}_{n} \star \mathcal{R}_{0}$ where $\mathcal{Q}_{k}$ (resp. $\mathcal{R}_{k}$ ) denotes the set of length- $k$ executions of $Q$ (resp. $R$ ). By generalising the previous rule to disjoint unions of $(n+1)$ terms, and using the fact that the number of elements of $\mathcal{Q}_{k} \star \mathcal{R}_{n-k}$ is $q_{k} r_{n-k}\left(\begin{array}{l}n \\ k\end{array}\right)$, one can select in which one of these terms to sample by drawing a random variable which is $k$ with probability $q_{k} r_{n-k}\left(\begin{array}{l}n \\ k\end{array}\right) / p_{n}$. Then it remains to sample a uniform element of $\mathcal{Q}_{k}$, a uniform element of $\mathcal{R}_{n-k}$ and a uniform shuffling of their labellings among the $\left(\begin{array}{l}n \\ k\end{array}\right)$ possibilities. This is described at line 7 of Algorithm 2. We do not detail the implementation of the shuffling function here, an optimal algorithm in terms of random bits consumption, can be found in 5 . As an example, consider the same programs as above: $Q=(a+(b \| c))$ and $R=$ $d^{\star}$. The number of length-3 executions of $(Q \| R)$ is $1 \cdot 1 \cdot\left(\begin{array}{l}3 \\ 1\end{array}\right)+2 \cdot 1 \cdot\left(\begin{array}{l}3 \\ 1\end{array}\right)=9$ using the decomposition $Q_{1} \star R_{2}+Q_{2} \star R_{1}$. Say $k=1$ is selected (with probability $1 / 3$ ), then the recursive calls to $(Q, 1)$ and $(R, 2)$ necessarily return $a$ and $d d$ and the SHUFFLE procedure must choose a shuffling uniformly between add, dad and $d d a$.

Sequential composition The case of the sequential composition is similar (see line 10 of Algorithm 21. We use the same kind of decomposition, using the Cartesian product $x$ in place of the labelled product $\star$. This has the consequence of removing the binomial coefficient in the formula for the generation of the $k$ random variable. Once $k$ is selected, we generate an execution of $Q_{k}$, an execution of $R_{n-k}$ and we concatenate the two.

Loop Finally, the case of the loop is a slight adaptation of the case of the sequential composition using the fact that the executions of $Q^{\star}$ are the executions 
of $\left(0+Q ; Q^{\star}\right)$. However, care must be taken to avoid issues related to doublecounting. More specifically, when sampling an execution of $\left(Q ; Q^{\star}\right)$ we must not choose an execution of length 0 for the left-hand-side $Q$. This is related to the same reason we had to specify the executions of $Q^{\star}$ as all the sequences of non-empty executions of $Q$. This is presented at line 13 of Algorithm 2, note that $k>0$. As an example, for sampling a length-3 execution in $(a+(b ; c))^{\star}$, one may select $k=1$ with probability $2 / 3$, which yields $a b c$ or aaa depending on the recursive call to $\left(Q^{\star}, 2\right)$ or $k=2$, with probability $1 / 3$, which yields $b c a$.

Generation of random variables We did not give details on how to generate the random variable $k$ for the parallel, sequential and loop case. Molinero showed in 20]19] that good performance can be achieved by using the so-called boustrophedonic order. For instance, in the case of the sequential composition $P=$ $(Q ; R)$, the idea is to generate a random integer $x$ in the interval $\llbracket 0 ; p_{n} \llbracket$ and to find the minimum number $\ell$ such that the sum of $\ell$ terms $q_{0} r_{n}+q_{n} r_{0}+q_{1} r_{n-1}+$ $q_{n-1} r_{1}+q_{2} r_{n-2}+\cdots$ (taken in this particular order) is greater than $x$. Then $k$ is such that the last term of this sum is $q_{k} r_{n-k}$.

Theorem 2. Using the boustrophedonic order, the complexity of the random generation of an execution of length $n$ in $P$ in terms of arithmetic operations on big integers is $O(n \cdot \min (\ln (n), h(P)))$ where $h(P)$ refers to the height of $P$ i.e. its maximum number of nested operators.

Contrary to the classical context of random generation in the context of analytic combinatorics (like in $13[1920$ ), the grammar enumerating the executions is not a constant but rather a parameter of the problem. Hence its size cannot be considered constant and the complexity analysis needs to be carefully crafted to take this variable into account.

Proof. The $O(n \ln (n))$ bound follows from Theorem 11 of 20. We obtain the other bound by refining the result of Theorem 12 from the same source. The combinatorial classes we are considering are built from the $\star, \times,+$ and $\operatorname{SeQ}(\cdot)$ operators without recursion, they hence fall under the scope of iterative classes for which Molinero proved a linear complexity in $n$. However the proof given in [20] does not give an explicit bound for the multiplicative constants, which actually depends on the size of the grammar and which we cannot consider constant in our context. Let $C(P, n)$ denote the cost of $\operatorname{UnIFExEC}(P, n)$ in terms of arithmetic operations on big integers. We show that $C(P, n) \leqslant \alpha n h(P)$ by induction for some constant $\alpha$ to be specified later.

- The base cases have a constant cost.

- The case of the choice only incurs a constant number $c$ of arithmetic operations in addition to the cost of the recursive calls. Hence $C(Q+R, n)$ is bounded by $c+\alpha \max (C(Q, n), C(R, n)) \leqslant c+\alpha n \max (h(Q), h(R))=$ $c+\alpha n(h(Q+R)-1)$ by induction. Thus, if $\alpha \geqslant c$, then $C(Q+R, n) \leqslant$ $\alpha \operatorname{nh}(Q+R)$. 
- The parallel composition case incurs a number of arithmetic operations of the form $c^{\prime} \min (k, n-k)$ where $k$ is the random variable generated using the boustrophedonic order technique. Hence $C(Q \| R, n)$ is bounded by $c^{\prime} \min (k, n-k)+C(Q, k)+C(R, n-k)$ and by induction by $c^{\prime} \min (k, n-$ $k)+\alpha k h(Q)+\alpha(n-k) h(R) \leqslant \alpha n h(Q \| R)+c^{\prime} \min (k, n-k)-\alpha n$. The last term on the right is bounded by 0 if $\alpha \geqslant c^{\prime}$.

- Sequential composition is treated using the same argument as for parallel composition.

- Finally, the loop must be handled by reasoning "globally" on the total number of unrollings. Say the loop $Q^{\star}$ is unrolled $r$ times. Then its cost $C\left(Q^{\star}, n\right)$ is bounded by $\sum_{i=1}^{r} c^{\prime} \min \left(k_{i}, k_{i+1}+\cdots+k_{r}\right)+\sum_{i=1}^{r+1} C\left(Q, k_{i}\right)$. The first sum is bounded by $c^{\prime} n$ and the second is bounded by induction by $\sum_{i=1}^{r+1} \alpha k_{i} h(Q)$ which simplified to $\alpha n h(Q)$. Hence, reusing the bound $\alpha \geqslant c^{\prime}$ and the fact that $h\left(Q^{\star}\right)=1+h(Q)$, we get $C\left(Q^{\star}, n\right) \leqslant \alpha n h\left(Q^{\star}\right)$ which terminates the proof.

\subsection{Execution prefixes}

The uniform sampler of executions described above provides one way of exploring the state space of a program, but it does not offer much flexibility. In this subsection we develop, as a complementary tool, a uniform random sampler of execution prefixes of given length. Note that this is different from using the previous algorithm until a length threshold $n$ because this would not yield uniform prefixes. An execution prefix is a sequence of evaluation steps as in Definition 2 but unlike an execution, its resulting program $P_{n}$ does not necessarily satisfy nullable $\left(P_{n}\right)$. We see this algorithm as an elementary building block for statistical exploration of the state-space, enabling a variety of different exploration strategies, possibly biased towards some areas of interest in the state-space of the program but in a controlled manner.

The idea here is to apply our previous algorithm to a new program pref $(P)$ defined inductively using Table 4. Note that pref $(P)$ (as well as its specification) can be implemented in linear space by using pointers to refer to the substructures of $P$.

Proposition 1. Let $P$ be an NFJ program. The executions of the pref $(P)$ are in one-to-one correspondence with the prefixes of executions of $P$.

Proof. The way execution prefixes are defined, the transformation is direct. We only discuss the cases where a $\mathcal{E}$ must be removed. In the case of the sequential composition, a prefix of execution of $P ; Q$ is either a prefix of $P$ or a complete execution of $P$ followed by a non-empty prefix of $Q$. It is important to only consider non-empty prefixes in order to avoid counting the complete executions of $P$ twice. In the case of the choice $P+Q$, we always subtract $\mathcal{E}$ from $\langle Q\rangle$ to avoid double-counting the empty prefix because all programs have it. Finally the case of the loop is a generalisation of the sequence: a prefix of $P^{\star}$ is made of any number of non-empty complete executions of $P$ followed by a non-empty prefix of $P$. 
Table 4. In the second column: the pref () transformation, mapping a program $P$ to a program whose executions are in correspondence with the prefixes of executions of $P$. In the third column: the combinatorial specification of the prefixes of $P$.

\begin{tabular}{lll}
\hline Program & Prefix program & Specification of the prefixes \\
$P$ & $\operatorname{pref}(P)$ & $\langle P\rangle$ \\
\hline 0 & 0 & $\mathcal{E}$ \\
$a$ & $0+a$ & $\mathcal{E}+\mathcal{Z}$ \\
$P \| Q$ & $(\operatorname{pref}(P) \| \operatorname{pref}(Q))$ & $\langle P\rangle \star\langle Q\rangle$ \\
$P ; Q$ & $\operatorname{pref}(P)+(P ; \operatorname{pref}(Q))$ & $\langle P\rangle+S(P) \times(\langle Q\rangle \backslash \mathcal{E})$ \\
$P+Q$ & $\operatorname{pref}(P)+\operatorname{pref}(Q)$ & $\langle P\rangle+(\langle Q\rangle \backslash \mathcal{E})$ \\
$P^{\star}$ & $P^{\star} ; \operatorname{pref}(P)$ & $\mathcal{E}+S\left(P^{\star}\right) \times(\langle P\rangle \backslash \mathcal{E})$ \\
\hline
\end{tabular}

As an example, using the notations $P_{0}=P_{1} ;(e+(f \| g))$, where $P_{1}=$ $\left(P_{2} \|(d+0)\right)^{\star}$ and $P_{2}=(a+(b \| c))^{\star}$, the specification of the prefixes of example program $P_{0}$ is given by:

$$
\begin{aligned}
& \left\langle P_{0}\right\rangle=\left\langle P_{1}\right\rangle+S\left(P_{1}\right) \times((\mathcal{E}+\mathcal{Z}+(\mathcal{E}+\mathcal{Z}) \star(\mathcal{E}+\mathcal{Z}) \backslash \mathcal{E}) \backslash \mathcal{E}) \\
& \left\langle P_{1}\right\rangle=\mathcal{E}+S\left(P_{1}\right) \times\left(\left\langle P_{2}\right\rangle \star(\mathcal{E}+\mathcal{Z}+\mathcal{E} \backslash \mathcal{E}) \backslash \mathcal{E}\right) \\
& \left\langle P_{2}\right\rangle=\mathcal{E}+S\left(P_{2}\right) \times((\mathcal{E}+\mathcal{Z}+(\mathcal{E}+\mathcal{Z}) \star(\mathcal{E}+\mathcal{Z}) \backslash \mathcal{E}) \backslash \mathcal{E})
\end{aligned}
$$

where $S\left(P_{1}\right)$ and $S\left(P_{2}\right)$ are not given but can be obtained as sub-terms of the specification $S\left(P_{0}\right)$ of the executions of $P_{0}$ given earlier.

Theorem 3. To sample uniformly a prefix of length $n$ in $P$ we sample uniformly a full execution in pref $(P)$. This has the same complexity as sampling an execution of $n$ up to a multiplicative constant.

The latter theorem is a consequence of Proposition 1. The complexity bound is obtained by showing that the height of $\operatorname{pref}(P)$ is at most twice the height of $P$. Another possibility of equal complexity would be to express directly the specification of the prefixes of $P$ without actually constructing the intermediate program. This specification is denoted $\langle P\rangle$ and is given in the third column of Table 4

\section{Experimental study}

In order to assess experimentally the efficiency of our method, in this section we put into use the algorithms presented in the paper and demonstrate that they can handle systems with a significantly large state space. We generated a few NFJ programs at random using a Boltzmann random generator. In its basic form, the Boltzmann sampler would generate a high number of loops and a large number of sub-terms of the form $P+0$ in the programs which we believe is not realistic so we tuned it using [4] so that the number of both types of nodes represent only $10 \%$ of the size of the program in expectation. We rely 
on the FLINT library (Fast Library for number theory [16]) to carry all the computations on polynomials except for the coloured product and the inversion, which we implemented ourselves in order to achieve the complexity exhibited in the previous section. The former was not provided natively by the library and the latter was feasible using FLINT's primitives but slow compared to the dedicated algorithm based on Newton iteration.

Note that besides the choice of the algorithms, we did not optimize our code for efficiency nor ran extensive tests on a big dataset, hence the numbers we give should be taken as a rough estimate of the performance of our algorithms. For the sake of reproducibility, the source code of our experiments is available on the companion repository ${ }^{8}$

\subsection{Preprocessing phase}

First, Table 5 gives the runtime of the preprocessing phase (Algorithm 1) that computes the generating functions of all the sub-terms of a program up to a given degree $n$. We measured this for programs of different sizes and for different values of $n$. Every measure was performed 7 times and we reported the median of these 7 values. The time reported is the CPU time as measured by C's clock function. The state-space column indicates the number of executions of length at most $n$ obtained by evaluating the polynomial with $z=1$. The figure on the right displays more data and focuses on the relation between the runtime of the preprocessing (on the $\mathrm{y}$ axis, in seconds) and the size of the state-space (the $\mathrm{x}$ axis is the $\log _{2}$ of the number of executions). Each line corresponds to a program and each point corresponds to a different value of $n$ for this program. Using a log-scale on both axis, this figure gives experimental "evidence" of a polynomial relation between the two. Besides the shape of the curves, the take-away here is that the preprocessing phase can be carried out for systems with a state-space of size $\approx 2^{18000}$ in a time of the order of one minute.

\subsection{Random generation}

We then measure the runtime of the random generator of executions and execution prefixes for the same programs. Every measure was performed 100 times and for each one we report the median of these values as well as the interquartile range (IQR) $)^{9}$, which gives an idea of the dispersion of the measures. We use these metrics rather than the mean and the variance to reduce the importance of extreme values and give a precise idea of what runtime the user should expect when running our sampler. A summary of the results is available in Table 6 .

\footnotetext{
${ }^{8}$ All the benchmarks were run on a standard laptop with an Intel Core i7-8665U and $32 \mathrm{G}$ of RAM running Ubuntu 19.10 with kernel version 5.3.0-46-generic. We used FLINT version 2.5.2 and GMP version 6.1.2.

9 The interquartile range of a set of measures is the difference between the third and the first quartiles. Compared with the value of the median, it gives a rough estimate of the dispersion of the measures.
} 
Table 5. On the left: runtime of the counting algorithm and size of the state-space (executions of length at most $n$ ) for programs of different sizes. On the right: plot of this runtime as a function of the $\log _{2}$ of the size of the state-space.

\begin{tabular}{lllr}
\hline$|P|$ & $n$ & $\#$ exec $^{\circ}$ & runtime \\
\hline 100 & 500 & $1.740825 \cdot 2^{1119}$ & $0.010 \mathrm{~s}$ \\
100 & 1000 & $1.073991 \cdot 2^{2235}$ & $0.037 \mathrm{~s}$ \\
100 & 3000 & $1.385924 \cdot 2^{6691}$ & $0.605 \mathrm{~s}$ \\
500 & 500 & $1.058776 \cdot 2^{1927}$ & $0.076 \mathrm{~s}$ \\
500 & 1000 & $1.081276 \cdot 2^{3832}$ & $0.462 \mathrm{~s}$ \\
500 & 3000 & $1.341591 \cdot 2^{11423}$ & $6.428 \mathrm{~s}$ \\
1000 & 500 & $1.473353 \cdot 2^{2330}$ & $0.159 \mathrm{~s}$ \\
1000 & 1000 & $1.044525 \cdot 2^{4712}$ & $0.874 \mathrm{~s}$ \\
1000 & 3000 & $1.092147 \cdot 2^{14181}$ & $13.488 \mathrm{~s}$ \\
2000 & 100 & $1.981851 \cdot 2^{410}$ & $0.012 \mathrm{~s}$ \\
2000 & 200 & $1.800651 \cdot 2^{926}$ & $0.049 \mathrm{~s}$ \\
2000 & 500 & $1.768618 \cdot 2^{2380}$ & $0.330 \mathrm{~s}$ \\
2000 & 1000 & $1.215440 \cdot 2^{4746}$ & $1.870 \mathrm{~s}$ \\
5000 & 500 & $1.607519 \cdot 2^{2923}$ & $0.897 \mathrm{~s}$ \\
5000 & 1000 & $1.469086 \cdot 2^{6016}$ & $5.434 \mathrm{~s}$ \\
5000 & 3000 & $1.226718 \cdot 2^{18116}$ & $75.649 \mathrm{~s}$ \\
\hline
\end{tabular}

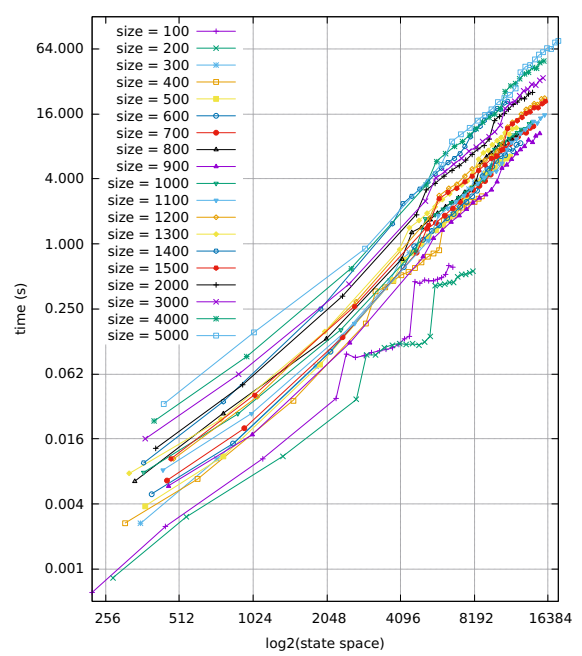

Interestingly, the number of executions and the numbers of execution prefixes are rather close. We have clues about the reasons behind this phenomenon which relate to the analytical properties of the generating functions of executions and prefixes. We will investigate this in the future but this is way beyond the scope of this article. As the numbers show, both random sampling procedures take a few milliseconds, even for rather large state-spaces. Here, the two state-space columns refer respectively to the number of executions and the number of prefixes of length exactly $n$. This is the cardinal of the set in which we sample a uniform element.

\subsection{Prefix covering}

This subsection presents an experimentation that highlights the importance of the uniform distribution for the purpose of state-space exploration. The setup is the following: consider a given NFJ program and randomly sample prefixes of given length $n$ of this program using two different algorithms:

- our random sampler which is globally uniform among all prefixes of length $n$;

- a "naive" sampler that repeatedly generates one execution step uniformly among the legal steps, until we get a length $n$ prefix. This strategy is called locally uniform or isotropic.

The question is: in average, how many random prefixes must be generated in order to discover a given proportion of the possible prefixes? This question 
Table 6. Median and interquartile range (IQR) of the runtime of the executions and prefixes samplers for various program sizes and object lengths.

\begin{tabular}{lllrrlrr}
\hline$|P|$ & $n$ & \# exec & UnIFEXEC & IQR & \# prefixes & UnIFPREFIX & IQR \\
\hline 100 & 500 & $1.370 \cdot 2^{1119}$ & $0.129 \mathrm{~ms}$ & $4 \mu \mathrm{s}$ & $1.841 \cdot 2^{1128}$ & $0.147 \mathrm{~ms}$ & $3 \mu \mathrm{s}$ \\
100 & 1000 & $1.690 \cdot 2^{2234}$ & $0.276 \mathrm{~ms}$ & $10 \mu \mathrm{s}$ & $1.124 \cdot 2^{2244}$ & $0.307 \mathrm{~ms}$ & $18 \mu \mathrm{s}$ \\
100 & 3000 & $1.090 \cdot 2^{6691}$ & $1.076 \mathrm{~ms}$ & $43 \mu \mathrm{s}$ & $1.439 \cdot 2^{6700}$ & $1.371 \mathrm{~ms}$ & $359 \mu \mathrm{s}$ \\
500 & 500 & $1.969 \cdot 2^{1926}$ & $0.218 \mathrm{~ms}$ & $5 \mu \mathrm{s}$ & $1.022 \cdot 2^{1997}$ & $0.281 \mathrm{~ms}$ & $12 \mu \mathrm{s}$ \\
500 & 1000 & $1.004 \cdot 2^{3832}$ & $0.563 \mathrm{~ms}$ & $21 \mu \mathrm{s}$ & $1.404 \cdot 2^{3901}$ & $0.688 \mathrm{~ms}$ & $33 \mu \mathrm{s}$ \\
500 & 3000 & $1.245 \cdot 2^{11423}$ & $3.718 \mathrm{~ms}$ & $203 \mu \mathrm{s}$ & $1.466 \cdot 2^{11492}$ & $4.005 \mathrm{~ms}$ & $274 \mu \mathrm{s}$ \\
1000 & 500 & $1.420 \cdot 2^{2330}$ & $0.301 \mathrm{~ms}$ & $10 \mu \mathrm{s}$ & $1.556 \cdot 2^{2411}$ & $0.352 \mathrm{~ms}$ & $19 \mu \mathrm{s}$ \\
1000 & 1000 & $1.005 \cdot 2^{4712}$ & $0.777 \mathrm{~ms}$ & $28 \mu \mathrm{s}$ & $1.293 \cdot 2^{4790}$ & $0.871 \mathrm{~ms}$ & $46 \mu \mathrm{s}$ \\
1000 & 3000 & $1.051 \cdot 2^{14181}$ & $4.829 \mathrm{~ms}$ & $481 \mu \mathrm{s}$ & $1.127 \cdot 2^{14259}$ & $5.307 \mathrm{~ms}$ & $569 \mu \mathrm{s}$ \\
2000 & 500 & $1.704 \cdot 2^{2380}$ & $0.308 \mathrm{~ms}$ & $14 \mu \mathrm{s}$ & $1.839 \cdot 2^{2484}$ & $0.416 \mathrm{~ms}$ & $10 \mu \mathrm{s}$ \\
2000 & 1000 & $1.169 \cdot 2^{4746}$ & $1.021 \mathrm{~ms}$ & $51 \mu \mathrm{s}$ & $1.482 \cdot 2^{4856}$ & $1.225 \mathrm{~ms}$ & $86 \mu \mathrm{s}$ \\
2000 & 3000 & $1.634 \cdot 2^{14120}$ & $7.291 \mathrm{~ms}$ & $1.2 \mathrm{~ms}$ & $1.921 \cdot 2^{14256}$ & $7.245 \mathrm{~ms}$ & $238 \mu \mathrm{s}$ \\
5000 & 500 & $1.589 \cdot 2^{2923}$ & $0.309 \mathrm{~ms}$ & $7 \mu \mathrm{s}$ & $1.933 \cdot 2^{3168}$ & $0.348 \mathrm{~ms}$ & $14 \mu \mathrm{s}$ \\
5000 & 1000 & $1.448 \cdot 2^{6016}$ & $0.898 \mathrm{~ms}$ & $43 \mu \mathrm{s}$ & $1.340 \cdot 2^{6231}$ & $1.027 \mathrm{~ms}$ & $41 \mu \mathrm{s}$ \\
5000 & 3000 & $1.208 \cdot 2^{18116}$ & $18.526 \mathrm{~ms}$ & $1.5 \mathrm{~ms}$ & $1.034 \cdot 2^{18324}$ & $21.478 \mathrm{~ms}$ & $1.2 \mathrm{~ms}$ \\
\hline
\end{tabular}

actually falls under the scope of the Coupon Collector Problem, which is treated in depth in [11. Table 7 gives numerical answers for both exploration strategies for a random NFJ program of size 25 and for a target coverage of $20 \%$ of the possible prefixes.

Expectedly the uniform strategy is faster but what is interesting to see is that the speedup compared to the isotropic method grows extremely fast. The more the state-space grows, the more the uniform approach is unavoidable.

Unfortunately, the formula given in 11 for the isotropic case involves the costly computation of power-sets which makes it impractical to give values for larger programs and prefix length. However, these small-size results already establish a clear difference between the two methods. It would be interesting to have theoretical bounds to quantify this explosion or to investigate more efficient ways to compute these values but this falls out of the scope of this article.

Table 7. Expected number of prefixes to be sampled to discover $20 \%$ of the prefixes of a random program of size 25 with either the isotropic or the uniform method.

\begin{tabular}{llllll}
\hline $\begin{array}{l}\text { Prefix length } \\
\text { \# prefixes }\end{array}$ & 1 & 2 & 3 & 4 & 5 \\
\hline Isotropic & 2.1 & 4.45 & 11.17 & 35.09 & $1.28 \cdot 10^{14}$ \\
Uniform & 2.1 & 3.18 & 6.57 & 13.26 & 27.69 \\
\hline Gain & $0 \%$ & $40 \%$ & $70 \%$ & $165 \%$ & $4.61 \cdot 10^{14} \%$ \\
\hline
\end{tabular}




\section{References}

1. Abbes, S., Mairesse, J.: Uniform generation in trace monoids. In: 40th International Symposium MFCS. pp. 63-75 (2015)

2. Arora, S., Barak, B.: Computational Complexity - A Modern Approach. Cambridge University Press (2009)

3. Basset, N., Mairesse, J., Soria, M.: Uniform sampling for networks of automata. In: 28th International Conference CONCUR (2017)

4. Bendkowski, M., Bodini, O., Dovgal, S.: Polynomial tuning of multiparametric combinatorial samplers, pp. 92-106 (2018)

5. Bodini, O., Dien, M., Genitrini, A., Peschanski, F.: Entropic Uniform Sampling of Linear Extensions in Series-Parallel Posets. In: 12th International Symposium CSR. pp. 71-84 (2017)

6. Bodini, O., Dien, M., Genitrini, A., Peschanski, F.: The Ordered and Colored Products in Analytic Combinatorics: Application to the Quantitative Study of Synchronizations in Concurrent Processes. In: 14th SIAM Meeting ANALCO. pp. 16-30 (2017)

7. Bodini, O., Genitrini, A., Peschanski, F.: The Combinatorics of Non-determinism. In: IARCS Annual Conference FSTTCS. vol. 24, pp. 425-436 (2013)

8. Bodini, O., Genitrini, A., Peschanski, F.: A Quantitative Study of Pure Parallel Processes. Electronic Journal of Combinatorics 23(1), P1.11, 39 pages (2016)

9. Bodini, O., Dien, M., Genitrini, A., Peschanski, F.: The Combinatorics of Barrier Synchronization. In: International Conference Petri Nets. pp. 386-405. Springer (2019)

10. Darrasse, A., Panagiotou, K., Roussel, O., Soria, M.: Boltzmann generation for regular languages with shuffle. In: GASCOM 2010 - Conference on random generation of combinatorial structures. Montréal, Canada (Sep 2010)

11. Flajolet, P., Gardy, D., Thimonier, L.: Birthday Paradox, Coupon Collectors, Caching Algorithms and Self-Organizing Search. D. A. Math. 39(3), 207-229 (1992)

12. Flajolet, P., Sedgewick, R.: Analytic Combinatorics. Cambridge University Press (2009)

13. Flajolet, P., Zimmermann, P., Cutsem, B.V.: A calculus for the random generation of combinatorial structures (1993)

14. Gaudel, M., Denise, A., Gouraud, S., Lassaigne, R., Oudinet, J., Peyronnet, S.: Coverage-biased random exploration of models. Electr. Notes Theor. Comput. Sci. 220(1), 3-14 (2008)

15. Grosu, R., Smolka, S.A.: Monte carlo model checking. In: 11th International Conference TACAS. pp. 271-286 (2005)

16. Hart, W., Johansson, F., Pancratz, S.: FLINT: Fast Library for Number Theory (2013), version 2.5.2, http://flintlib.org

17. Koomen, C.J.: Calculus of Communicating Systems, pp. 11-26. Springer US, Boston, MA (1991)

18. Krob, D., Mairesse, J., Michos, I.: On the average parallelism in trace monoids. In: 19th Annual STACS. pp. 477-488 (2002)

19. Martínez, C., Molinero, X.: An experimental study of unranking algorithms. In: Ribeiro, C.C., Martins, S.L. (eds.) Experimental and Efficient Algorithms. pp. 326-340. Springer Berlin Heidelberg, Berlin, Heidelberg (2004)

20. Molinero, X.: Ordered generation of classes of combinatorial structures. Ph.D. thesis, Universitat Politècnica de Catalunya (Oct 2005) 
21. Oudinet, J., Denise, A., Gaudel, M.C., Lassaigne, R., Peyronnet, S.: Uniform Monte-Carlo Model Checking. In: 14th International Conference FASE (2011)

22. Pivoteau, C., Salvy, B., Soria, M.: Algorithms for combinatorial structures: Wellfounded systems and Newton iterations. Journal of Combinatorial Theory, Series A 119, 1711-1773 (2012)

23. Valiant, L.G.: The complexity of computing the permanent. Theor. Comput. Sci. 8, 189-201 (1979) 